\title{
INFLUENCE OF STOKES DRIFT ON SALT WEDGE INTRUSION EVALUATED USING FULLY-NONLINEAR AND STRONGLY-DISPERSIVE WAVE EQUATIONS
}

\author{
Keisuke Nakayama ${ }^{1}$, Tetsuya Shintani ${ }^{2}$, Taro Kakinuma ${ }^{3}$, Yasuyuki Maruya ${ }^{4}$, Yoshinori Yonome $^{1}$ \\ Mitsuaki Yonemoto ${ }^{5}$ and Isao Nakauchi ${ }^{5}$

\begin{abstract}
This paper describes the influence of surface waves on salt-wedge intrusion in terms of radiation stress. Radiation stress may increase salt-wedge intrusion when surface waves propagate up a river. This study thus aims to reveal the effect of radiation stress on the distance of salt-wedge intrusion by using fully nonlinear strongly dispersive internal wave equations and three-dimensional numerical computation model, Fantom3D. Fully nonlinear strongly dispersive internal wave model reveals the possibility that large radiation stress is induced near the river mouth and increases the distance of salt-wedge intrusion. Three-dimensional numerical model also demonstrates that there is a significant difference in the intrusion distance by taking into account radiation stress.
\end{abstract}

Keywords: variable principle; radiation stress; salt-wedge intrusion

\section{Introduction}

In estuaries, mixing of fresh water and sea water is one of the significant factors sustaining and maintaining sound bio-diversity. Many brackish lakes exist along the coastal area of the Okhotsk Sea, which have rich marine products, such as salmon, trout, pond smelt, scallop, and so on. In recent years, it is demonstrated that ecological system may be deteriorated due to the change in mixing process, such as distance of salt-wedge intrusion which may be due to climate change (Maruya et al. 2010).

In the previous studies regarding salt-wedge intrusion, many investigations have been done for understanding the mechanisms based on tidal and wind effect (Kuwajima et al. 1993; Ikenaga et al. 1998; Ikenaga et al. 1999; Yoshikawa and Watanabe 2006; Yoshikawa et al. 2007). One of the previous studies proposed the theory for estimating the distance of salt-wedge intrusion using the difference between sea-water and lake-water levels. Sakuma et al. (2010) revealed that wind waves also enhance the distance of salt-wedge intrusion although the detailed theory for the estimation of the intrusion distance was not proposed.

This study thus aims to clarify the effect of wind waves on the distance of salt-wedge intrusion based on radiation stress by using fully-nonlinear and strongly-dispersive wave equations (Kakinuma 2001). The evaluation of the distance of salt-wedge intrusion was conducted by using threedimensional environmental numerical model, Fantom3D (Shintani and Nakayama 2009).

\section{Fully nonlinear and strongly dispersive wave equations}

The FSI equations are derived based on the theory proposed by Luke (1967), and the details are described in Nakayama \& Kakinuma (2010). The FSI equations can be applied to more general cases because the mild-slope assumption is not used. Furthermore, since the size of the target area is about $2000 \mathrm{~m}$ x $5 \mathrm{~m}$ (horizontal size x vertical size), it is difficult to carry out 2D numerical computation due to the expensive run-time cost. Therefore, fully-nonlinear and strongly dispersive wave equations attempted to be applied in terms of the accuracy of wave motions and the run-time cost.

Wave equations in a two-layer system with an irrotational flow field are obtained by expanding velocity potential using power function (Fig. 1).

$$
\begin{gathered}
\mathbf{u}_{i}=\nabla \phi_{i} \text { and } w_{i}=\partial \phi_{i} / \partial z \\
\phi_{i}(x, z, t)=\sum_{\alpha=0}^{N-1} Z_{i, \alpha}\left\{z, h_{i}(x)\right\} f_{i, \alpha}(x, t) \equiv Z_{i, \alpha} f_{i, \alpha}
\end{gathered}
$$

\footnotetext{
${ }^{1}$ Department of Civil and Environmental Engineering, Kitami Institute of Technology, Koen-cho 165, Kitami city, 0908507, Japan

2 Department of Civil and Environmental Engineering, Tokyo Metropolitan University, 1-1 Minami-Osawa, Hachioji city, 192-0397, Japan

${ }^{3}$ Department of Ocean Civil Engineering, Kagoshima University, Korimoto 1-2-24, Kagoshima city, 890-8580, Japan

${ }^{4}$ Graduate School of Civil Engineering, Kitami Institute of Technology, Koen-cho 165, Kitami city, 090-8507, Japan

${ }^{5}$ Hokkaido Regional Development Bureau, Shin-matchi 2-6-1, Abashiri city, 093-8544, Japan
} 


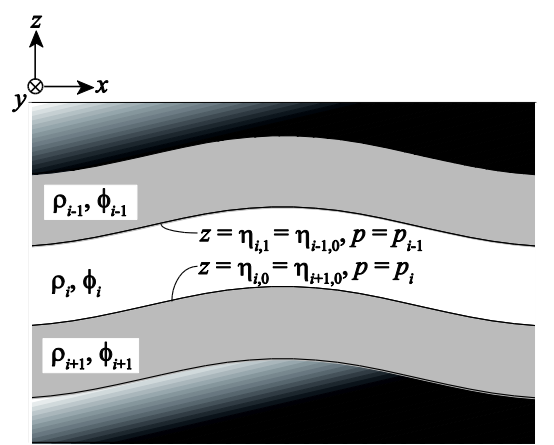

Figure 1. Multilayer fluid system

where $\nabla$ is a partial differential operator in the horizontal plane, i.e., $\nabla=(\partial / \partial x, \partial / \partial y), N$ is the number of vertically distributed functions and the sum rule of product is adopted for subscript $\alpha, f_{i, \alpha}$ is the weight for the $i^{\text {th }}$-layer.

The definition of the vertical profile function as Eq. 3 yields upper and lower layer equations.

$$
Z_{i, \alpha}=Z^{\alpha}
$$

Upper layer equations

$$
\begin{aligned}
& \eta^{a} \frac{\partial \eta}{\partial t}+\frac{1}{\alpha+\beta+1} \nabla\left(\eta^{\alpha+\beta+1} \nabla f_{1, \beta}\right)-\frac{\alpha \beta}{\alpha+\beta-1} \eta^{\alpha+\beta-1} f_{1, \beta}=0 \\
& \eta^{\beta} \frac{\partial f_{1, \beta}}{\partial t}+\frac{1}{2} \eta^{\beta+\gamma} \nabla f_{1, \beta} \nabla f_{1, \gamma}+\frac{\beta \gamma}{2} \eta^{\beta+\gamma-2} f_{1, \beta} f_{1, \gamma}+g \eta+\frac{p_{1}}{\rho_{1}}=0
\end{aligned}
$$

Lower layer equations

$$
\begin{aligned}
& \eta^{a} \frac{\partial \eta}{\partial t}+\frac{1}{\alpha+\beta+1} \nabla\left(\left(\eta^{\alpha+\beta+1}-b^{\alpha+\beta+1}\right) \nabla f_{2, \beta}\right\} \\
& -\frac{\alpha \beta}{\alpha+\beta-1}\left(\eta^{\alpha+\beta-1}-b^{\alpha+\beta-1}\right) f_{2, \beta}=0 \\
& \eta^{\beta} \frac{\partial f_{2, \beta}}{\partial t}+\frac{1}{2} \eta^{\beta+\gamma} \nabla f_{2, \beta} \nabla f_{2, \gamma} \\
& +\frac{\beta \gamma}{2} \eta^{\beta+\gamma-2} f_{2, \beta} f_{2, \gamma}+g \eta+\frac{p_{1}+\left(\rho_{2}-\rho_{1}\right) g h_{1}}{\rho_{2}}=0
\end{aligned}
$$

In the FSI equations, velocity potential is defined using the expansion of power function, which means that the more the number of $\mathrm{N}$ is, the higher the accuracy of computation reaches. When $\mathrm{N}=1$, the FSI equations are the same as the shallow water equations. When $\mathrm{N}$ is between 2 and 3 , the FSI equations are found to be the same as the Boussinesq equations by Peregrine (1967) for waves with weak nonlinearity and dispersion in relatively shallow water (Kakinuma \& Nakayama, 2007). Although the equations are for a two-layer system, it is possible to apply the equations into surface waves by taking the upper and lower layer as air and water.

\section{Field observation data in River Abashiri}

Lake Abashiri is located along the coastal area of the Okhotsk Sea which is connected to the ocean with the river length of $7 \mathrm{~km}$, and salt-wedge intrusion often occurs when the sea-water level is higher than lake water level (Fig. 2). Base flow discharge of River Abashiri is about $10 \mathrm{~m}^{3} / \mathrm{s}$, which is much smaller than the discharge induced by tidal effect at the river mouth, $60 \mathrm{~m}^{3} / \mathrm{s}$. Since the average water depth of River Abashiri is about $2 \mathrm{~m}$, well-mixed density front is revealed to intrude from the ocean to the upstream.

To understand the attenuation of wave amplitude along the river, field observation was carried out at five stations by using wave hunter $\Sigma$ (Fig. 3). The field observation was conducted from the 7 th of November of 2007 to the 12th of December of 2007, in which the maximum significant wave height was found to be more than $3.0 \mathrm{~m}$ at the offshore when strong wind, more than $10 \mathrm{~m} / \mathrm{s}$, was measured. 

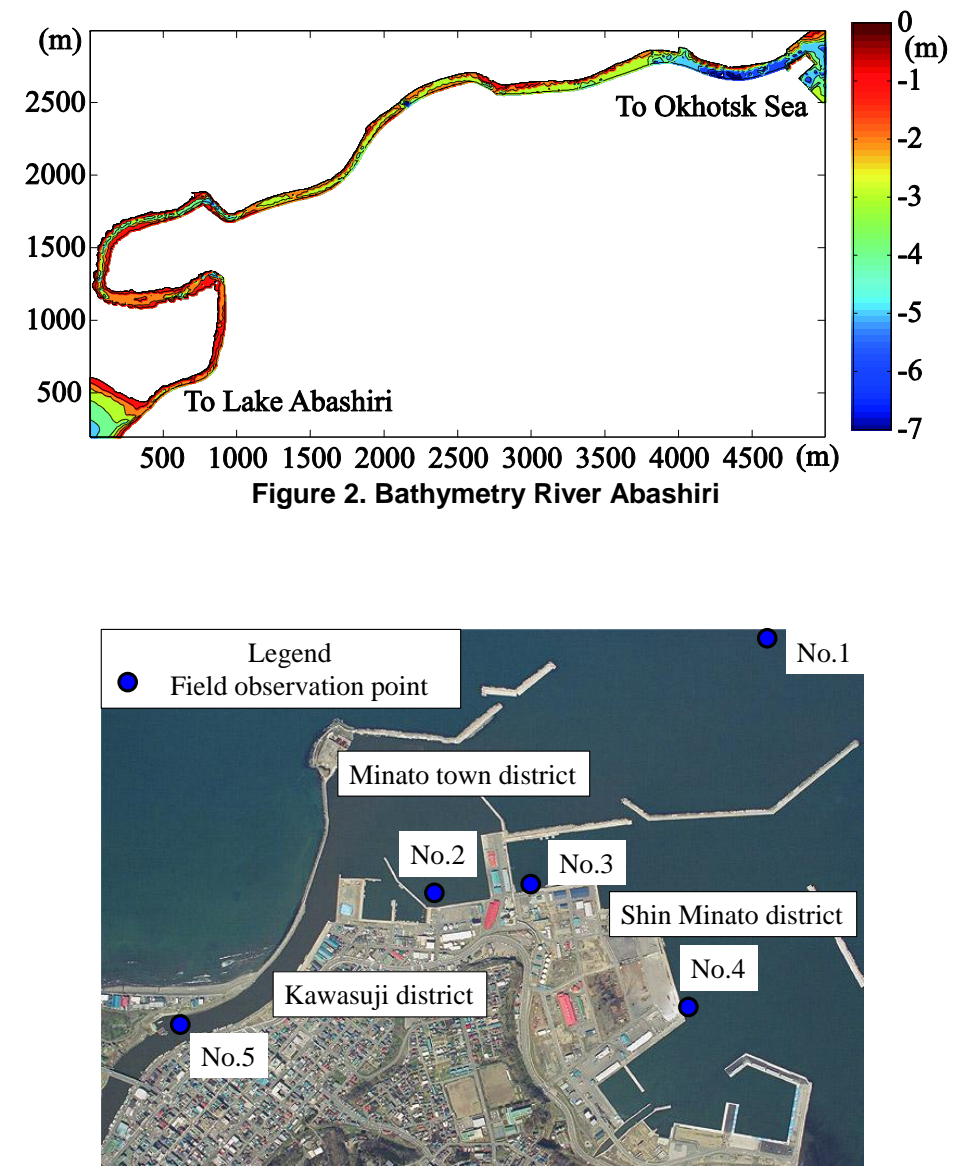

Figure 3. Field Observation stations of wave around Abashiri harbors
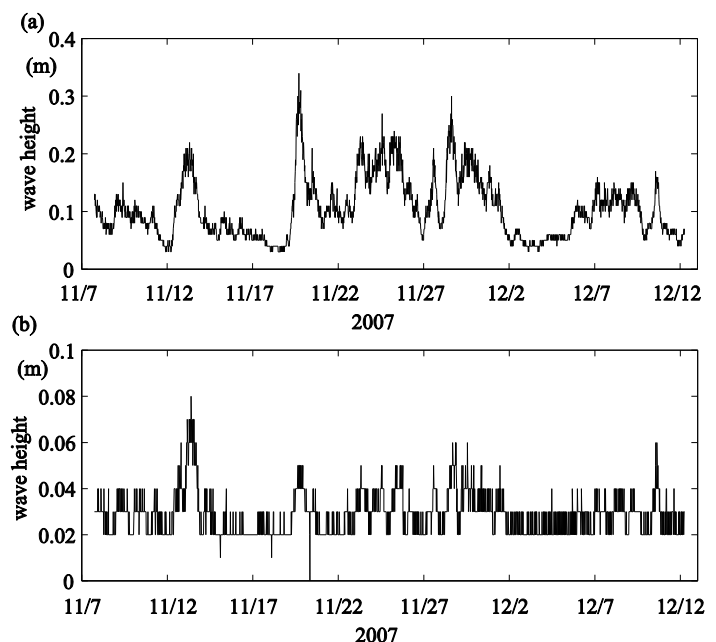

Figure 4. Significant wave height at (a) No.2 and (b) No.5

Field observation data at No.2 and No.5 was used as the data at the river mouth and $600 \mathrm{~m}$ from the river mouth.

When the significant wave height at the offshore was more than $3.0 \mathrm{~m}$, the significant wave height and period at No.2 were $0.19 \mathrm{~m}$ to $0.34 \mathrm{~m}$ and $10.6 \mathrm{~s}$ to $13.6 \mathrm{~s}$ (Fig. 4). Also, the significant wave height and period at No.5 were $0.03 \mathrm{~m}$ to $0.05 \mathrm{~m}$ and $6.8 \mathrm{~s}$ to $8.5 \mathrm{~s}$. In River Abashiri, the ratio of water depth and wave length is more than $1 / 10$, which means that dispersion relation should be included in the analysis of radiation stress. 


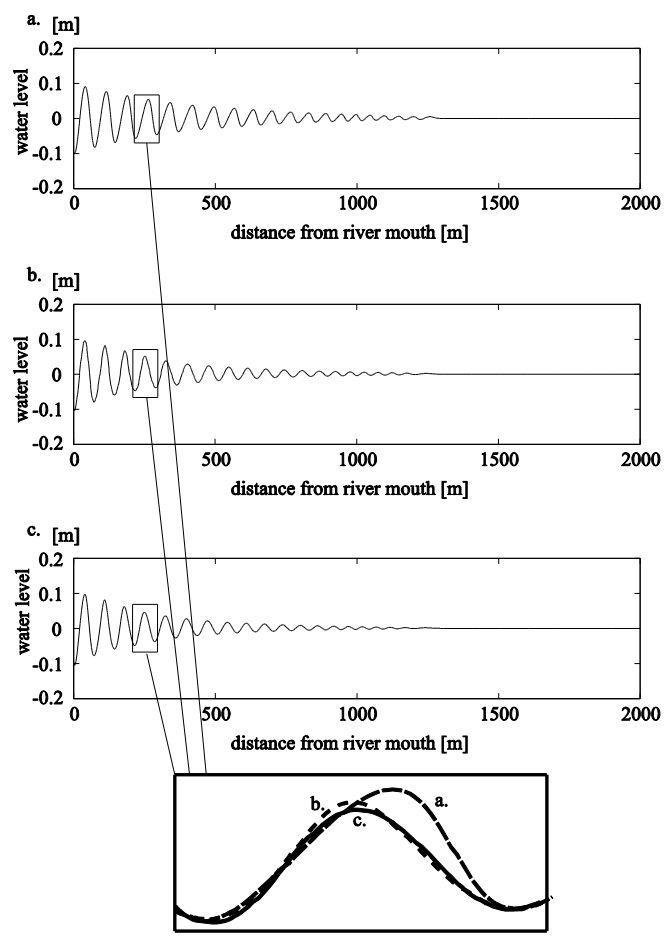

Figure 5. Spatial distribution of waves. (a) $N=1$, (b) $N=2$ and (c) $N=3$
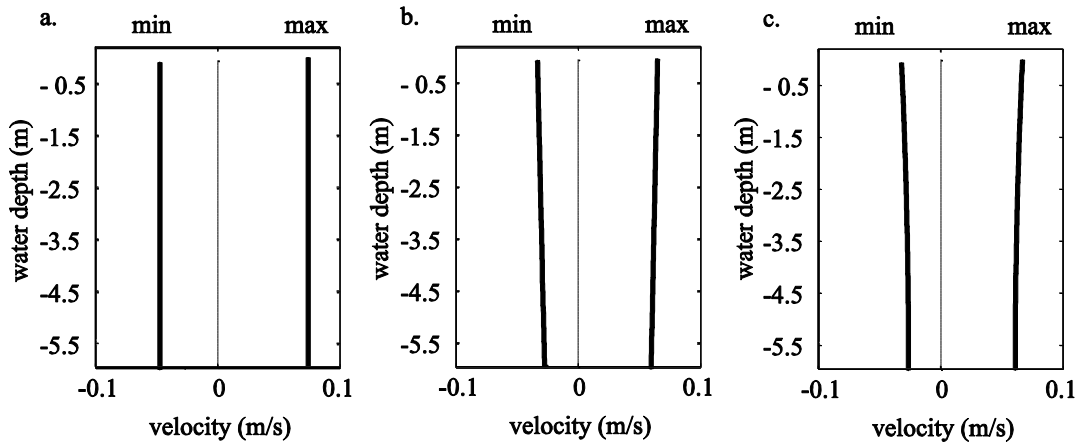

Figure 6. Vertical profile of maximum and minimum horizontal velocity at the distance of $300 \mathrm{~m}$ from the river mouth (a) $N=1$, (b) $N=2$ and (c) $N=3$

\section{Evaluation of radiation stress}

Fully nonlinear and strongly dispersive wave equations are applied for the evaluation of radiation stress along River Abashiri from the river mouth to $2000 \mathrm{~m}$ from the river mouth. According to the field observation data, the significant wave height is found to be about $0.2 \mathrm{~m}$ and $0.03 \mathrm{~m}$ at the river mouth and at $600 \mathrm{~m}$ from the river mouth. In the analysis, $N$ is considered up to 3 (Fig. 5). The computed waves show good agreements with the measured waves both in amplitude and wavelength when $N=3$. Cnoidal waves are revealed to occur when $N=3$ compared to $N=1$, which is supported by Nakayama and Kakinuma (2010). Therefore, $N=3$ or at least $N=2$ is found to be needed in the analysis.

To clarify the effect of dispersion relation, vertical profile of horizontal velocity was computed at $300 \mathrm{~m}$ from the river mouth (Fig. 6). It is confirmed from the results of $N=3$ and $N=2$ that the difference of horizontal velocity at the water surface and bottom is more than $10 \%$ compared to the maximum absolute velocity. The difference is caused by dispersion relation, which also suggests the necessity of the usage of that $N$ is more than 1 .

For the estimation of additional energy from waves into salt-wedge intrusion, radiation stress was computed by using Eq. 8 . 

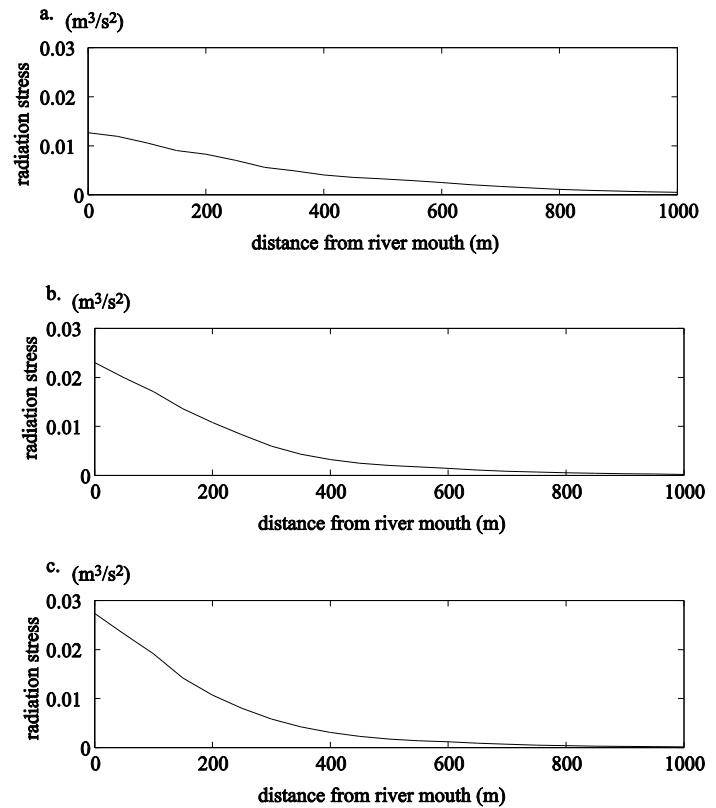

Figure 7. Radiation stress from river mouth (a) $N=1$, (b) $N=2$ and (c) $N=3$
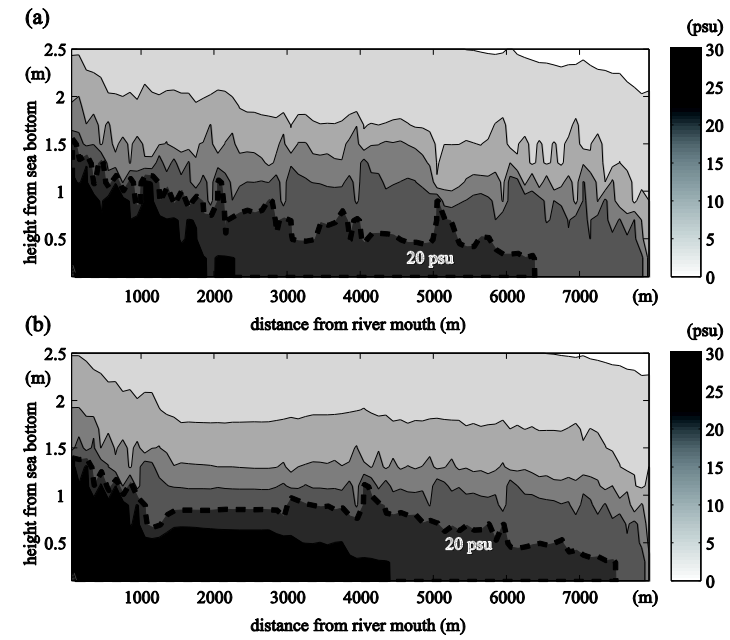

Figure 8. Spatial distribution of salinity (a) without radiation stress and (b) with radiation stress.

$$
S_{x x}=\overline{\int_{-b}^{-h_{1}+\eta}\left(\rho_{2} u_{2}{ }^{2}+p\right) d z}-\frac{1}{2} \rho_{2} g h_{2}{ }^{2}
$$

Although there is no great difference between $N=3$ and $N=2$ in the wave shape and vertical profile of horizontal velocity, the maximum value of radiation stress at the river mouth is found to increase with the increase in $N$ (Fig. 7). The radiation stress from $N=3$ is $20 \%$ more than $N=2$. In contrast to the maximum value at the river mouth, the decrease rate in radiation stress along the river is found to be larger when $N=3$ compared to $N=1$. This result also supports the necessity of higher accuracy wave model, such as $N=3$.

\section{Salt-wedge intrusion and radiation stress}

To clarify the effect of wave energy on salt-wedge intrusion, object-oriented three-dimensional numerical model was applied, Fantom3D (Shintani and Nakayama, 2009). Computational domain is 8 $\mathrm{km} \times 2 \mathrm{~m} \times 2 \mathrm{~m}$ with the size of mesh, $10 \mathrm{~m} \times 1 \mathrm{~m} \times 0.2 \mathrm{~m}$. To confirm the effect of waves, narrow computational domain is used. Time step is $10 \mathrm{~s}$ and the total computational time is 30 days. Bottom 
gradient is zero and vertically uniform horizontal velocity is given at the upstream end of the computational domain. The addition of radiation stress in the three-dimensional model is done by giving vertically uniform external force in the momentum equations which is obtained from Fig. 7.

Computational results show the fluctuation of the position of density front. Spectrum analysis reveals the maximum dominant fluctuation period is about 4 days. Therefore, 4 days average of density distribution is computed for making the comparisons with and without radiation stress (Fig. 8). It is obvious that the distance of density front with radiation stress is $1000 \mathrm{~m}$ more than without radiation stress. Also, density near water surface at the river mouth is higher with radiation stress rather than without radiation stress, which may enhance the distance of density front.

\section{Conclusions}

Fully nonlinear and strongly dispersive wave equations and Fantom3D are applied to investigate the effect of wave energy on salt-wedge intrusion. The following conclusions are obtained:

1. Fully nonlinear and strongly dispersive wave equations demonstrate that radiation stress is less estimated when $N=1$ or 2 , which suggests the necessity of the usage of high accuracy wave model, such as $N$ is more than 2 .

2. Fantom3D reveals that the distance of density front is likely to be enhanced by radiation stress, which is induced by wind energy.

\section{REFERENCES}

Ikenaga H., J. Ohtsuka, D. Misawa, K. Uchijima, K. Kashiyama, and T. Yamada. 1998. Study on the characteristics of salinity intrusion and change of salt wedge in Abashiri River, Annual Journal of Hydraulic Engineering, JSCE, 42, 775-780.

Ikenaga H., K. Mukouyama, S. Ohshima, and T. Yamada. 1999. Prediction of the long term behaviors of the density interface in a brackish lake, Journal of Hydraulic, Coastal and Environmental Engineering, JSCE, 628, 77-96.

Kakinuma T. 2001. Math calculation of behavior of internal wave in permeability beach, Proceedings of Coastal Engineering, JSCE, 48, 146-150.

Kakinuma T., and K. Nakayama. 2007. Numerical simulation of internal waves using a set of fully nonlinear internal wave equations, Annual Journal of Hydraulic Engineering, JSCE, 51, 169-174.

Kuwajima T., K. Takahashi, S. Miyajima, M. Hirano, and T. Yamada. 1993. Long and short term behavior of Lake Abashiri with two layers of fresh and salt water, Proceedings of Hydraulic Engineering, JSCE, 37, 305-312.

Luke, J.C. 1967. A variational principle for a fluid with a free surface, Journal of Fluid Mechanics, 27, 395-397. doi:10.1017/S0022112067000412.

Maruya Y., K. Nakayama, D. Horimatsu, S. Yonome, and M. Yonemoto. 2010. Influence evaluation of wind stress and inflow from river on stratification in Lake Abashiri, Annual Journal of Hydraulic Engineering, JSCE, 54, 1393-1398.

Nakayama K., and T. Kakinuma. 2010. Internal waves in a two-layer system using fully nonlinear internal-wave equations, International Journal for Numerical Methods in Fluids, 62:5, 574-590. doi: 10.1002/fld.2037.

Peregrine, D.H. 1967. Long waves on a beach, Journal of Fluid Mechanics Digital Archive, 27:4, 815827. doi: $10.1017 /$ S0022112067002605.

Sakuma N., K. Nakayama, T. Kakinuma, and T. Shintani. 2010. Influence of wind waves on salt wedge intrusion in Abashiri River, Proceedings of Hokkaido chapter of the Japan society of Civil Engineers, JSCE, B-52.

Shintani T., and K. Nakayama. 2009. Development and verification of object-oriented environment hydrodynamic model, Annual Journal of Hydraulic Engineering, JSCE, 53, 1267-1272.

Yoshikawa Y., and Y. Watanabe. 2006. Field observation on salinity intrusion at ice-covered rivers, Advances in River Engineering, JSCE, 12, 157-162.

Yoshikawa Y., H. Yasuda, and Y. Watanabe. 2007. Impact of a mesh structure on salinity intrusion in Abashiri River, Advances in River Engineering, JSCE, 13, 7-12. 